\title{
Long term treatment of chronic Lyme arthritis with benzathine penicillin
}

\author{
Marco A Cimmino, Silvano Accardo
}

\begin{abstract}
The cases are reported of two patients with chronic Lyme arthritis resistant to the recommended antibiotic regimens who were cured by long term treatment with benzathine penicillin. It is suggested that the sustained therapeutic levels of penicillin were effective either by the inhibition of germ replication or by lysis of the spirochaetes when they were leaving their sanctuaries.
\end{abstract}

(Ann Rheum Dis 1992; 51: 1007-1008)

The standard treatment for the early stages of Lyme borreliosis consists of a short cycle of tetracycline or phenoxymethyl penicillin. ${ }^{1}$ In cases of persistent infection associated with stage III manifestations such as chronic arthritis, acrodermatitis chronica atrophicans and encephalitis, two week cycles of intravenous ceftriaxone $^{2}$ or of penicillin G (benzylpenicillin) ${ }^{3}$ are recommended. These regimens, however, are occasionally ineffective $e^{4}$ and the question of which treatment strategy should be used in these patients remains unresolved.

This paper reports the cases of two patients with chronic arthritis due to Borrelia burgdorferi infection who were cured by long term treatment with benzathine penicillin after the failure of treatment with standard antibiotics.

\section{Case reports}

\section{PATIENT}

In January 1985 a 36 year old woman living in an area endemic for Lyme disease ${ }^{5}$ was admitted to this hospital with fever, intermittent macular rash, and symmetrical arthritis of her knees and wrists. On physical examination cervical and inguinal lymphadenomegaly, mild splenomegaly, and pharyngitis were found. Laboratory tests showed high levels of acute phase reactants but were negative for IgM rheumatoid factor and antinuclear antibodies. HLA tissue typing showed the presence of the DR4 antigen. IgG antibodies against $B$ burgdorferi were determined by indirect immunofluorescence after absorption with Treponema phagedenis to avoid cross reactions with $T$ pallidum. Their titre was $1 / 256$. The patient was treated with intravenous penicillin $G\left(20 \times 10^{6} \mathrm{U}\right.$ over 12 days $)$ and prednisone $(25 \mathrm{mg} /$ day $)$. Her symptoms improved and she was discharged while receiving a daily regimen of tetracycline $(200 \mathrm{mg})$, prednisone $(25 \mathrm{mg})$, hydroxychloroquine $(400$ $\mathrm{mg}$ ), and diclofenac (100 $\mathrm{mg}$ ). Her symptoms flared up when the prednisone dose was tapered. The patient was subsequently treated irregularly by her family doctor with non-steroidal antiinflammatory drugs (NSAIDs) and tetracycline.

In May 1986 synovectomy of the right wrist was performed. The specimen showed chronic synovitis, but no attempt was made to identify or culture spirochaetes. A radiogram of the carpal bones showed bone erosion. The patient was readmitted to hospital with persisting symptoms and signs of inflammation. A second course of penicillin $\mathrm{G}$ was given, but it was ineffective. Three months later benzathine penicillin was given at a dose of $1.2 \times 10^{6}$ $\mathrm{U} /$ week. This treatment was continued for six months. At the end of 1986 the patient was completely asymptomatic with negative (1/32) serum titres for $B$ burgdorferi. In January 1991 she remained healthy with normal haematological parameters.

\section{PATIENT 2}

In the summer of 1985 a 36 year old woman developed a skin rash consistent with erythema migrans a few days after being bitten by a tick in the right popliteal area. The rash lasted for two weeks and was followed one month later by a stiff neck, headache, diffuse arthralgia, and arthritis of the right knee. A diagnosis of rheumatoid arthritis was made and NSAIDs were given with no effect. Cryoglobulins, circulating immune complexes, and IgM rheumatoid factor were not present in serum samples and the erythrocyte sedimentation rate was within normal limits.

In October 1987 the patient was examined at this clinic because of chronic synovitis of the right knee. The white blood cell count in the synovial fluid was $7 \cdot 2 \times 10^{9} / 1$ ( $72 \%$ neutrophils). A biopsy sample obtained by arthroscopy showed a hypertrophic synovial membrane with vascular proliferation and marked infiltration of lymphocytes and plasma cells. A single borrelialike spirochaete was seen in the stroma by Warthin-Starry silver impregnation. The IgG titre against $B$ burgdorferi was $1 / 512$ in the serum sample and $1 / 256$ in the synovial fluid sample. Using an enzyme linked immunosorbent assay (ELISA) the absorbance of a sonicate of the IRS strain of the spirochaete was found to be 1.716 in the serum sample and 1.304 in the synovial fluid sample (normal value less than $0 \cdot 3$ ). The two samples reacted with western blotting to at least nine bacterial proteins including the $21,30,34,41,60$, and 100 kilodalton proteins. The HLA antigen DR2 was present.

The patient was treated with minocycline ( $200 \mathrm{mg} / 10$ days) and with NSAIDs with only a 
slight improvement of her symptoms. Two cycles of intramuscular ceftriaxone ( $2 \mathrm{~g} / 10$ days) were subsequently given in December 1987 and March 1988 but five months later the patient had not recovered from the arthritis. Benzathine penicillin treatment was started at this time at a dose of $1.2 \times 10^{6} \mathrm{U} /$ week, which was decreased to $1 \cdot 2 \times 10^{6} \mathrm{U} / 20$ days two months later when the patient became pregnant. The arthritis improved during the otherwise uneventful pregnancy and at delivery (August 1989) the patient was asymptomatic with antibody titres of $1 / 64$. The baby was healthy with no clinical signs of Lyme disease. In January 1991 both mother and child were in good health.

\section{Discussion}

Several explanations have been suggested for the occasional lack of response to the standard antibiotic treatment of chronic Lyme arthritis. Spirochaetes may persist for years in organs such as the spleen ${ }^{6}$ or the synovial membrane, or may find an intracellular sanctuary. ${ }^{7} \mathrm{~A}$ nonspecific reaction to cell wall components of $B$ burgdorferi has been suggested to sustain the arthritis even in the absence of live spirochaetes. ${ }^{8}$ Resistance to antibotic treatment has been associated with the presence of the HLA-DR4 antigen. ${ }^{9}$

Long term treatment with benzathine penicillin was effective in treating chronic Lyme disease in these two patients who had not responded to repeated cycles of ceftriaxone or penicillin. It is possible that recovery of the arthritis after the initial treatment was simply delayed because of the discussed non-specific response. This is contradicted, however, by the protracted duration of arthritis, which lasted three and five months after the withdrawal of antibiotics. Successful treatment is commonly defined as the resolution of joint inflammation within three months of the completion of treatment. Short courses of benzathine penicillin have been used to treat Lyme arthritis ${ }^{4}$ but only $35 \%$ of patients had a complete resolution of symptoms. We believe that the sustained therapeutic concentrations of circulating drug that are characteristic of benzathine penicillin treatment were effective in the long term in eradicating the infection, either by inhibition of germ replication or by lysis of the spirochaetes during the phase when they are temporarily leaving their sanctuaries. These anecdotal observations indicate the need for further studies on the efficacy of long term treatment with benzathine penicillin of refractory chronic Lyme arthritis.

1 Steere A C, Hutchinson G J, Rahn D W, et al. Treatment of the early manifestations of Lyme disease. Ann Intern Med 1983; 39: 22-6.

2 Dattwyler R J, Halperin J J, Pass H, Luft B J. Ceftriaxone as effective therapy in refractory Lyme disease. $\mathcal{J}$ Infect $D$ is 1987; 155: 1322-5.

3 Steere A C. Lyme disease. N Engl f Med 1989; 321: 586-96.

4 Steere A C, Green I, Schoen R T, et al. Successful parenteral penicillin therapy of established Lyme arthritis. $N$ Engl $\mathcal{f}$ Med 1985; 312: 869-74.

5 Cimmino M A, Trevisan G, Fumarola D, Crovato F. Lyme arthritis: clinical findings in Italian patients. In: Stanek G, ed. Lyme borreliosis II, Zbl Bakt. Suppl 18. New York: Fisher, 1989: 256-60.

6 Cimmino M A, Azzolini A, Tobia F, Pesce C M. Spirochaetes in the spleen of a patient with chronic Lyme disease. $A m \mathcal{F}$ Clin Pathol 1989; 91: 95-7.

$7 \mathrm{Ma} \mathrm{Y}$, Sturrock A, Weis J J. Intracellular localization of Borrelia burgdorferi within human endothelial cells. Infect Immun 1991; 59: 671-8.

8 Beck G, Benach J L, Habicht G S. Isolation, preliminary chemical characterisation, and biological activity of Borrelia burgdorferi peptidoglycan. Biochem Biophys Res Commun 1990; 167: 89-95.

9 Steere A C, Dwyer E, Winchester R. Association of chronic Lyme arthritis with HLA-DR4 and HLA-DR2 alleles. N Engl f Med 1990; 323: 219-23. 